\title{
SUPPLÉMENT GRAPHIQUE
}

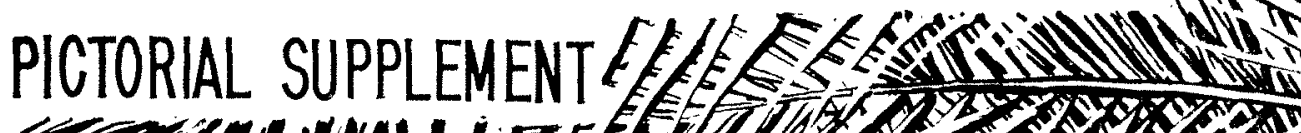
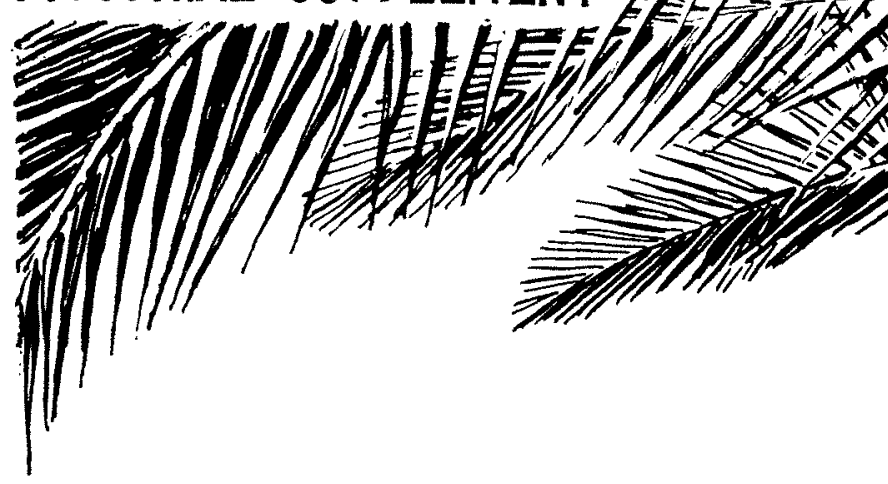

LA

CONDUITE

D'ORAN
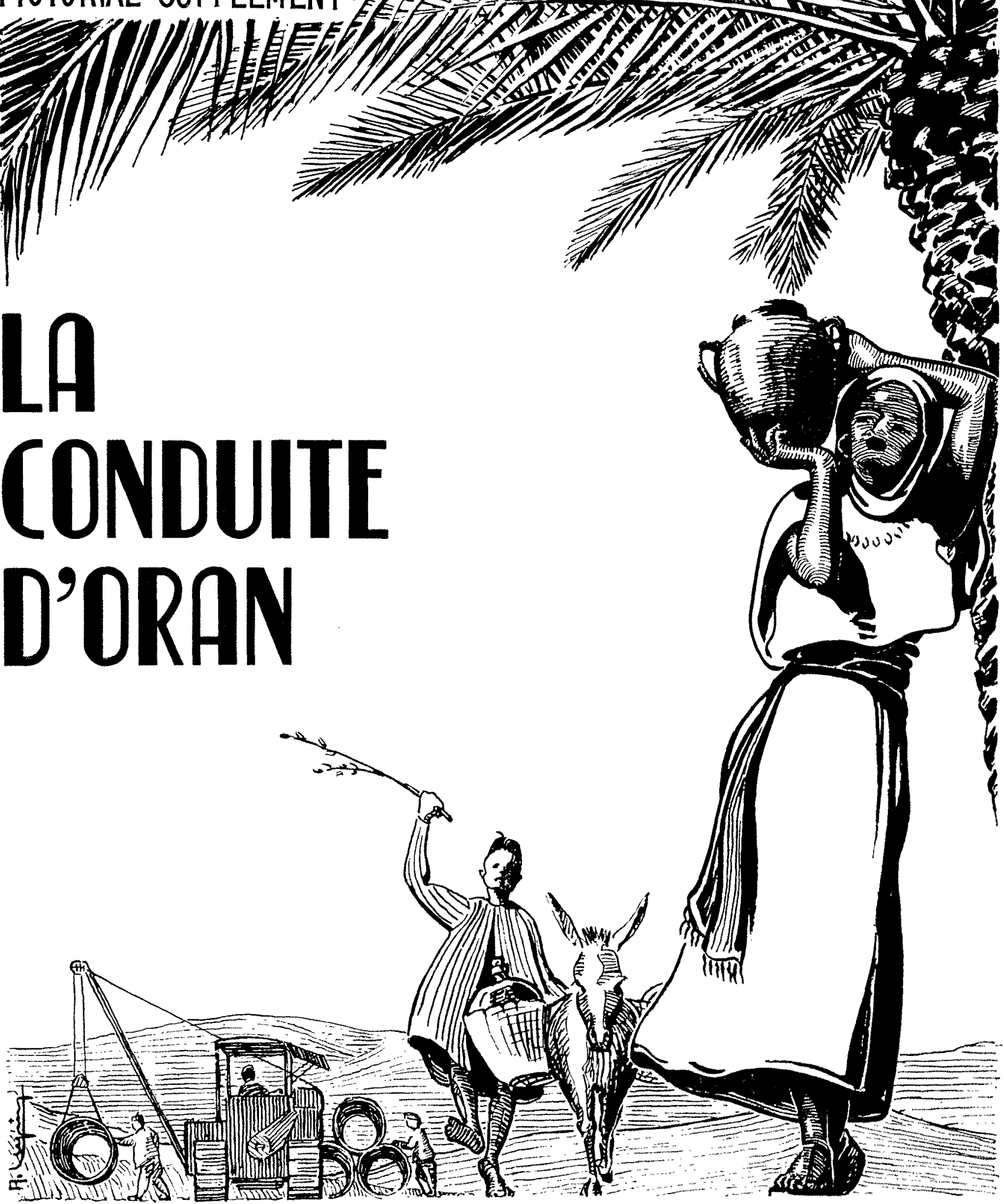
JUSQU'A L'AMENAGEMENT DE LA CONDUITE D'ORAN dont io mise en service est actuellement terminée, la ville d'Oran étart alimentée en eau d'une manière bien insuffisante, aussi bien en qualité qu'en quan-

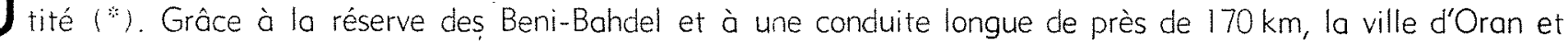
le port de Mers-el-Kébir bénéficient maintenant d'un débit de $60.000 \mathrm{~m} \% /$ jour, soit environ 200 litres par habitant sur la base de 300.000 habitants.

L'ensemble des ourrages consiste principalement an:

- Une retenue de 63 millions de $m^{3}$ d'eau créée par le barrage des Beni-Bahdel;

- Un souterrain de $11,4 \mathrm{~km}$ de longueur à écoulement libre;

- Un bassin de compensation de $80.000 \mathrm{~m}^{3}$ à Bou Hallou;

- Une station de filtration équipée pour clarifier et préchlorer $100000 \mathrm{~m}$ : par jour;

- Une conduite gravitaire de quelques $170 \mathrm{~km}$ de long, d'un diamètre de $1,10 \mathrm{~m}$, formée d'éléments de $7 \mathrm{~m}$ en béton précontraint, préfabriqués, et amenant les eaux de la cote 600 à la cote 175 environ;

- Des ouvrages d'art (brise-charges, passerelles de franchissement, souterrains) destinés à assurer le fonctionnement hydroulique de la conduite ou son passage dans les zones topographiquement difficiles;

- Des centrales électriques, situées le long du tracé, destinées à turbiner les débits transportés par la conduite;

(*) Ce texte sinspire lorgement de lo communicotion prósentée ou Congrès des Gronds Borrages de NevDehli (janvier 1951) par les Entreprises Socomon, EGTH Chobol et Neyrpic (Lobors. toire Douphinos diHydroulique).

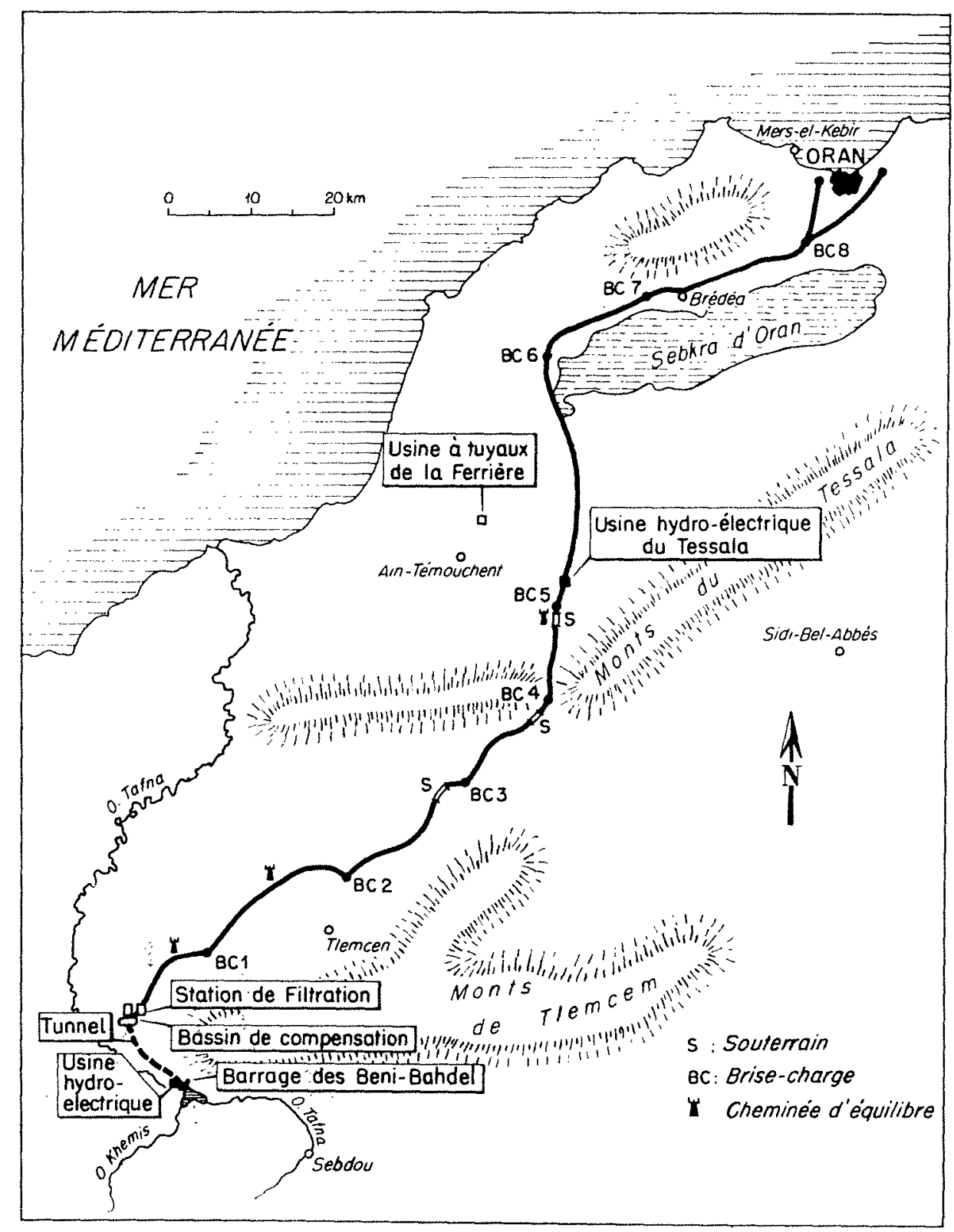

Répartition des ouvrages principaux le long de la conduite 


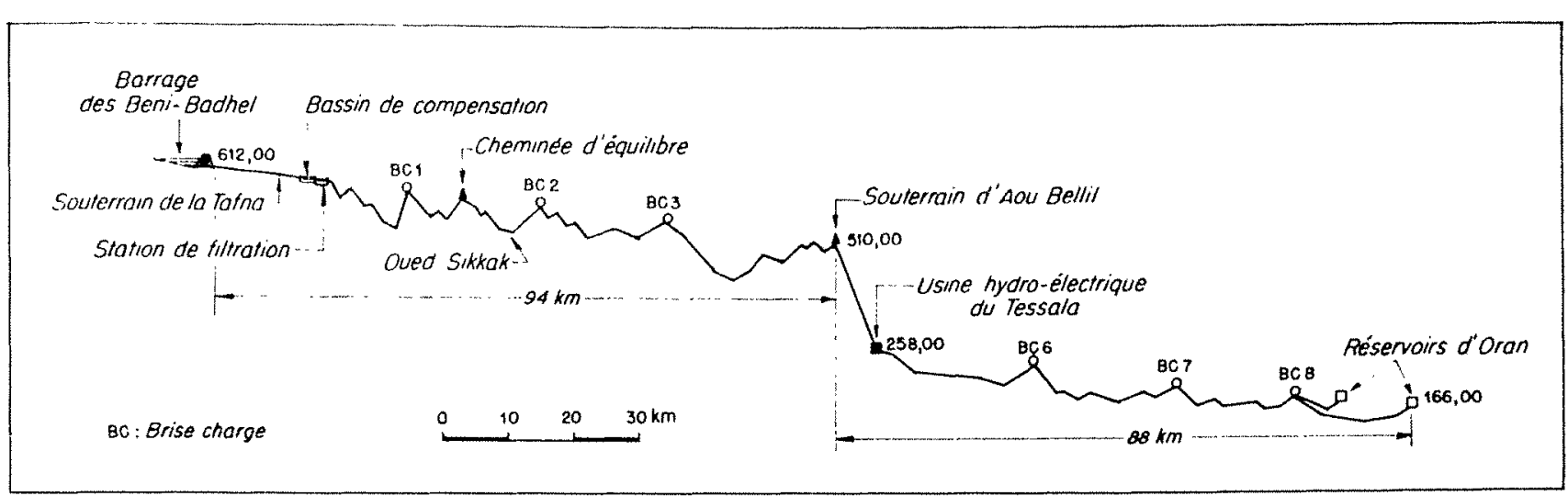

\section{Profil en long.}

- Une station de stérilisation en fin de canalisation, avant la distribution à Oran et Mers-el-Kébir;

- Des réservoirs ovant la distribution.

La caractéristique essentiellement originale de l'aménagement consiste dans la régularisation des débits qui, sur $170 \mathrm{~km}$ de longueur et plus, est automatiquement assurée par la demande de l'aval. Du bassin de compensation de Bou Hallou à la distribution d'eau en ville, sans intervention d'énergie extérieure ni appel à d'autres phénomènes qu'à l'hydraulique, les débits sont réglés en fonction de ce qu'utilisent au moment même les divers usagers. Lo transmission de la demande aussi bien le long de la conduite qu'à travers la station de filtration, s'effectue au moyen de vannes à flotteur situées, ò l'aval des sections, en tous les points où s'opère une rupture de charge et où règne une surface libre. Ces vannes y maintiennent le niveau constant, ouvrant s'il tend à baisser et inversement. Grâce à cet équipement, les pertes d'eau inutilisées sont lotalement éliminées; la conduite, toujours pleine, n'est plus soumise aux oscillations en masse ni oux coups de bélier dùs à la présence d'air entrainé et mal evacué. Cette technique est réalisée pour la première fois à Oran sur une aussi grande échelle.

\section{LE BARRAGE DES BENI-BAHDEL}

Il est, pour son tronçon principal, du type à voûtes multiples, de sections semi-circulaires, à épaisseur consrante dans une section droite. Ses dimensions principales sont les suivantes:

- Longueur totale $350 \mathrm{~m}$;

- Hauteur au milieu du thalweg: $55 \mathrm{~m}$ (couronnement à la cote 654,25);

- Houteur totale y compris les fondations: $70 \mathrm{~m}$.

La partie centrale, sur une longueur de $220 \mathrm{~m}$, comprend 11 voûtes de $20 \mathrm{~m}$ de diamètre, dont l'axe est incliné suivant un fruit de 0,95. Les deux extrémités latérales, sur une longueur de $80 \mathrm{~m}$ sur la rive gauche et de $50 \mathrm{~m}$ sur la rive droite, sont constituées par deux tronçons de barrage-poids.

Cet ouvrage principal est complété par deux petits barroges à voûtes multiples, barrant deux cols dont les altitudes sont inférieures au niveau maximum de la retenue.

Le premier de ces ouvrages, dit « digue du col Nord » o une hauteur moyenne de $15 \mathrm{~m}$ et une longueur de $220 \mathrm{~m}$. L'outre dit « digue du col de la Route » a une hauteur de $15 \mathrm{~m}$ et une longueur de $450 \mathrm{~m}$. La partie centrale de la digue du col de la Route est aménagée en évacuateur permettant de passer un débit de $1.000 \mathrm{~m}^{3} / \mathrm{s}$ avec une lame d'eau de $0,50 \mathrm{~m}$. Cet évacuateur est constitué par une série de 20 goulottes déversantes dites en «bec de canard » en raison de leur forme.

Les aménogements annexes du barrage principal comprennent:

- Un trop-plein susceptible d'évacuer $200 \mathrm{~m}^{3} / \mathrm{s}$. - Une vidange de fond. - Une prise d'eau. 


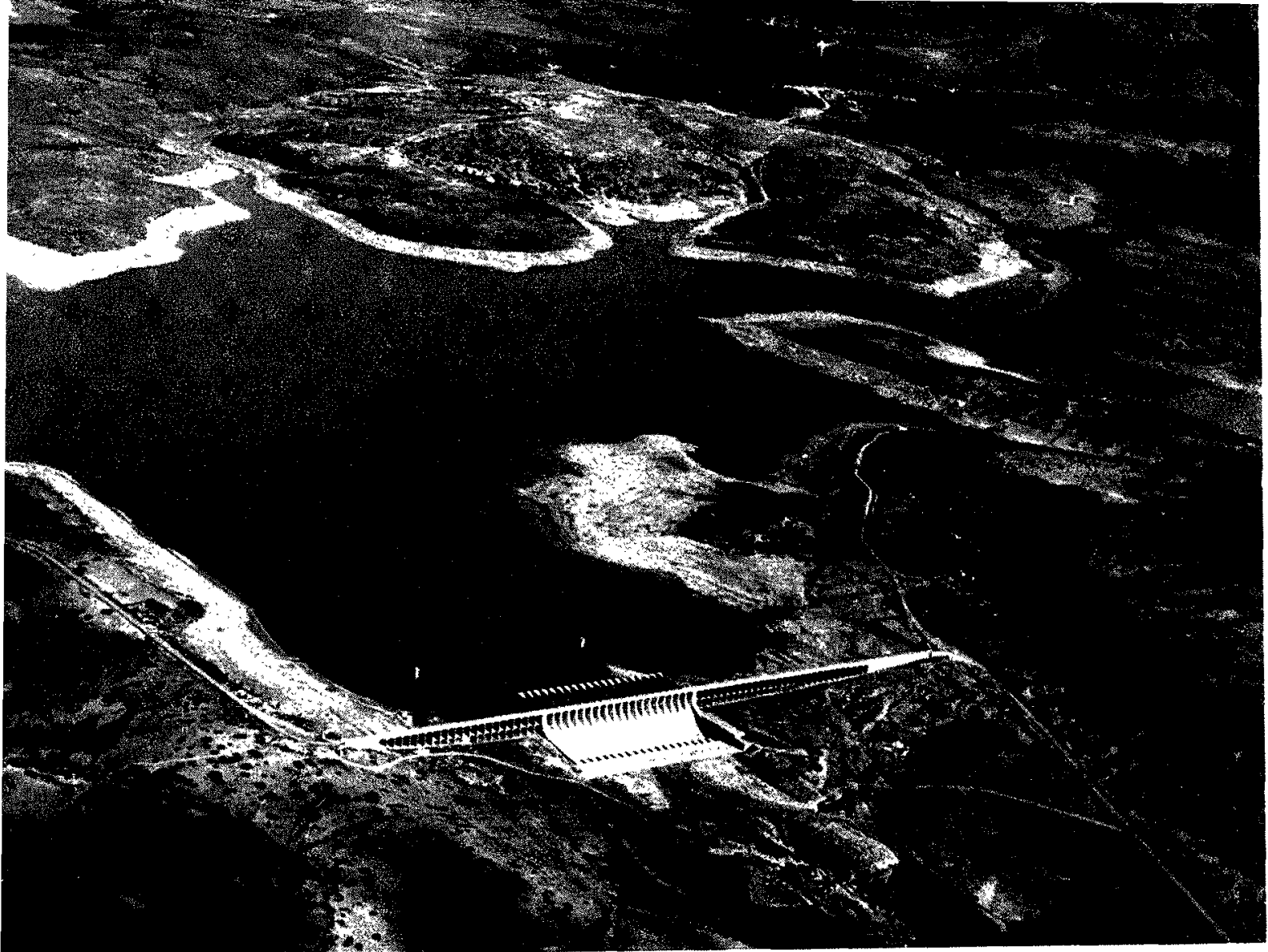

Cliche: Socomatn

Retenue des Béni-Bahdel.

Digue du col de la route

et évacuateur principal de crues.

Retenue des Béni-Bahdel.

Barrage principal.

Cliche fammenom-tienard

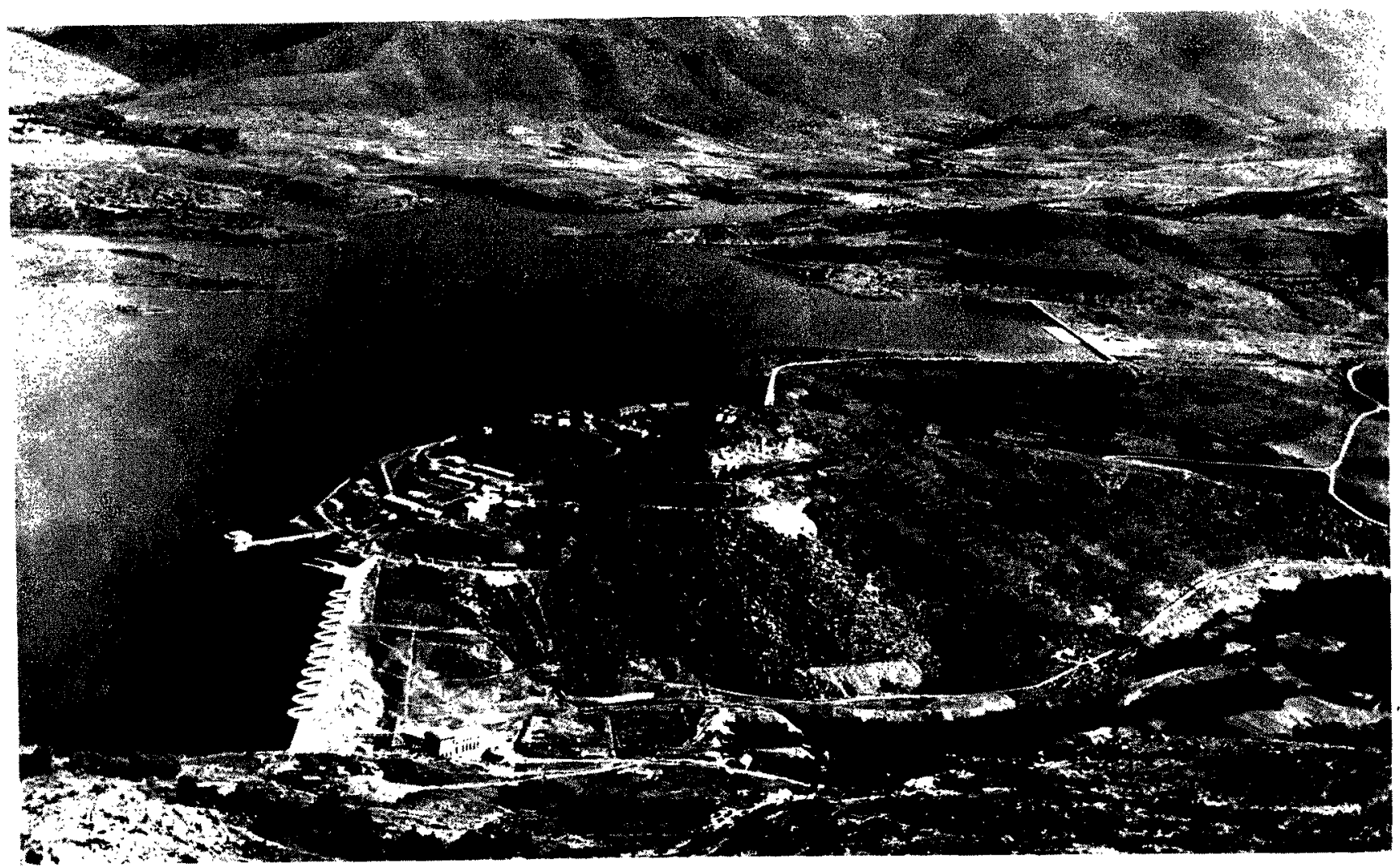


Clichin Campenon-Pernard

Voûtes du barrage principal pendant leur exécution vues d'amont

Digue du col de la route

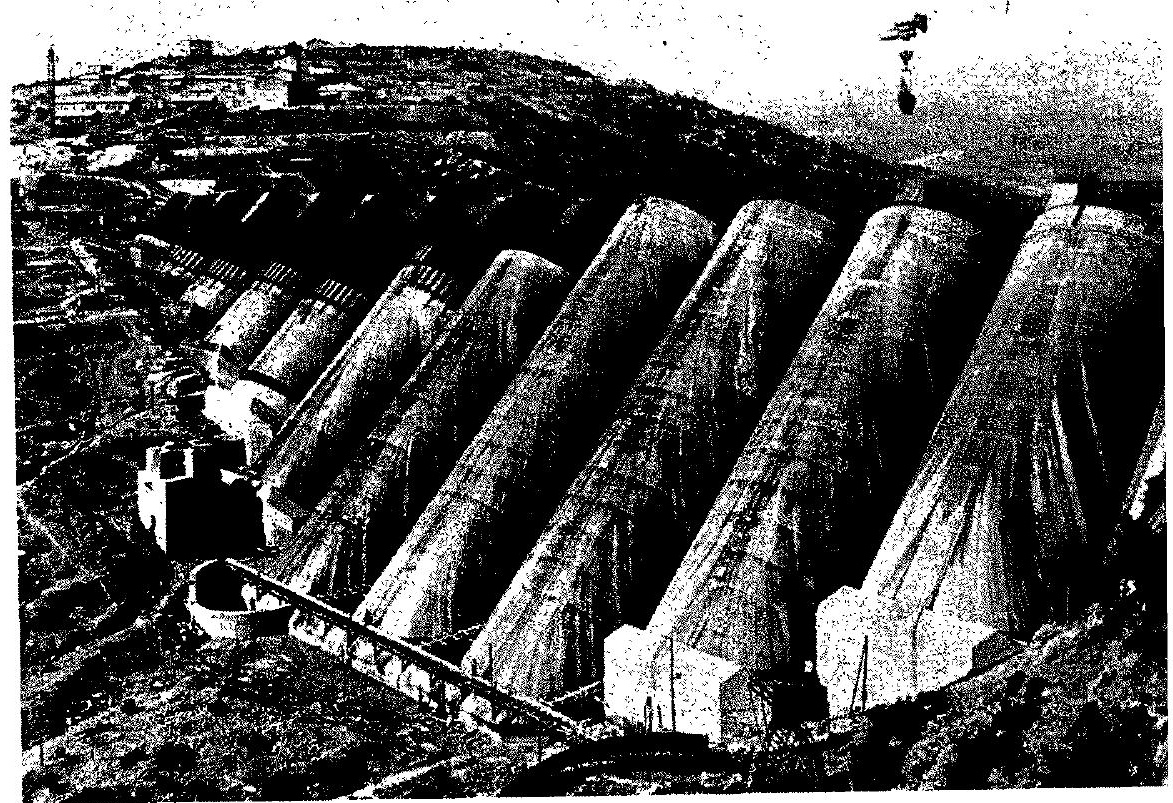

et évacuateur principal de crues.

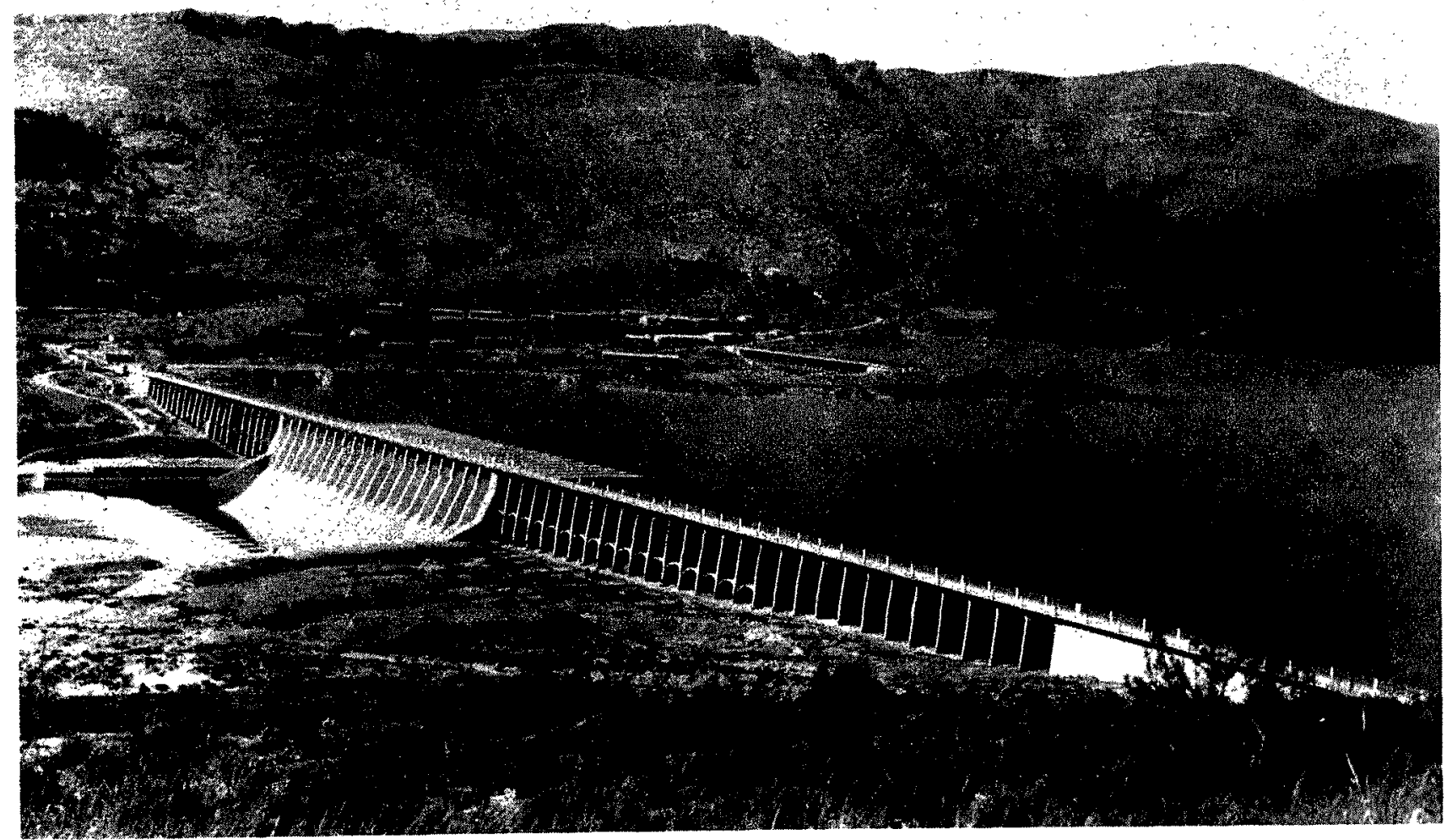

Barrage principal en construction.

vu d'aval

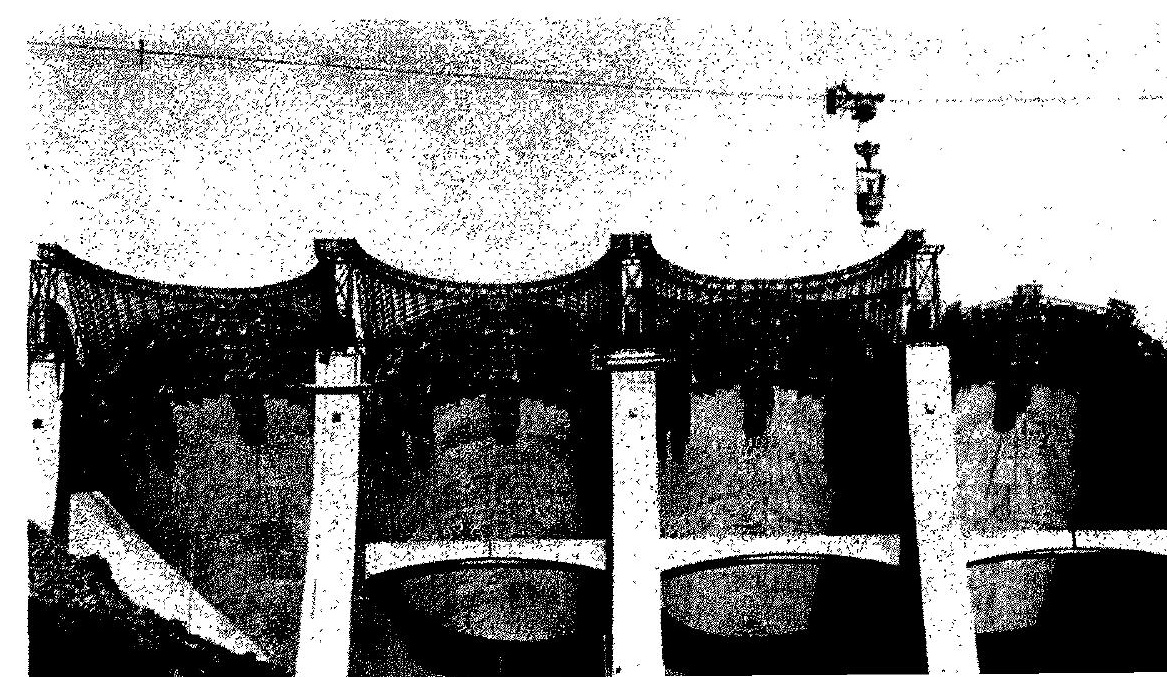


Au pied du borrage est instollée une usine hydro-ćlectrique destinée ò turbiner en pointe toutes les eaux lâchées du barrage. Son équipement, susceptible d'absorbor $10 \mathrm{~m}^{3} / \mathrm{s}$ sous $37 \mathrm{~m}$ de chule, comprend deux groupes Francis à axe vertical et un alternateur de $2.500 \mathrm{kVA}-5.000 \mathrm{~V}$.

\section{SOUTERRAIN DE LA TAFNA}

11 assure l'écoulement de $6 \mathrm{~m}^{3} / \mathrm{s}$ entre l'usine de pied de barrage et le bassin de compensation de Bou Hallou. Lusine pouvant turbiner $10 \mathrm{~m}^{3} / \mathrm{s}$ les 180 premiers mètres du souterrain sont susceptibles de les évacuer

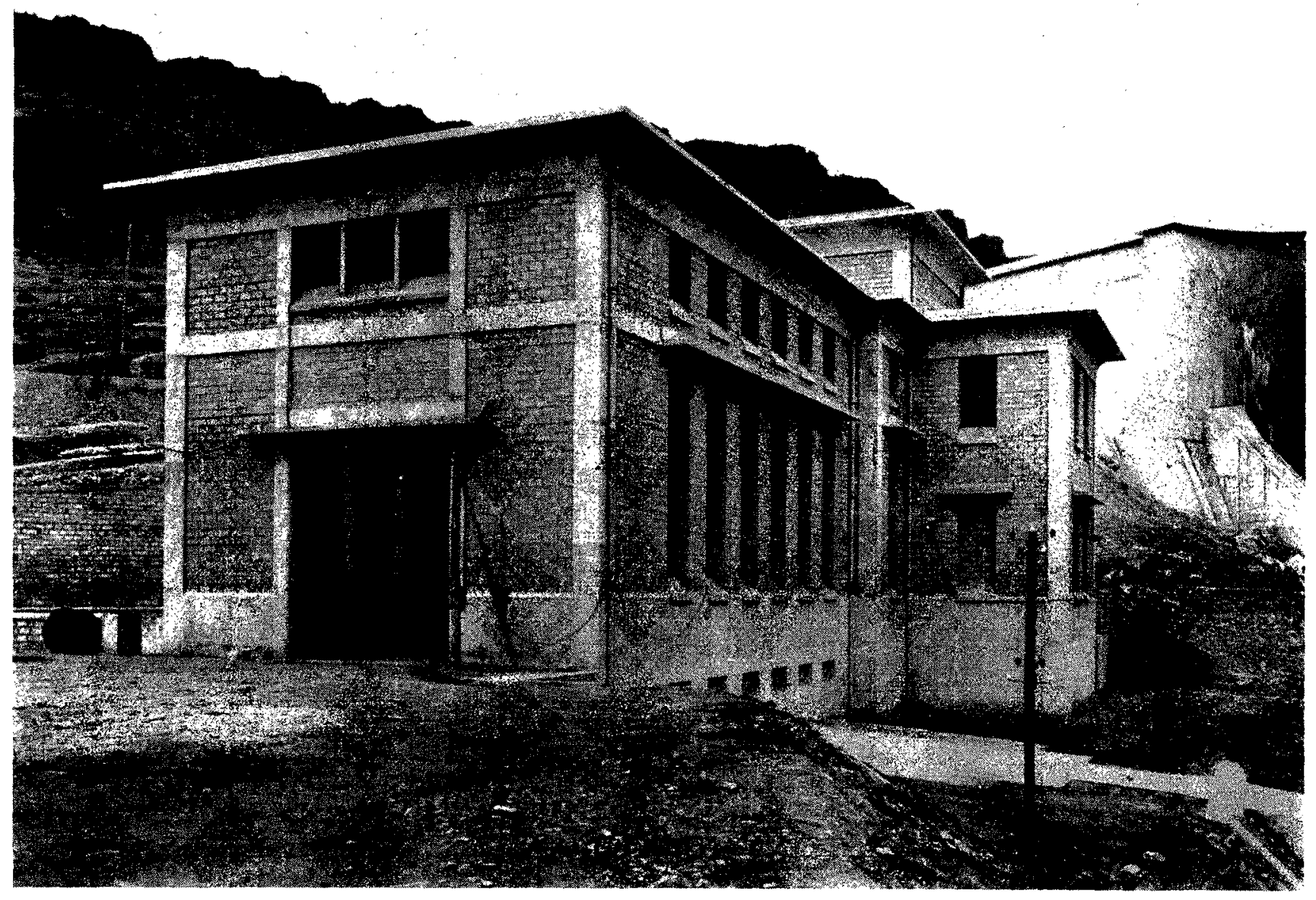

Usine de pied de barrage.

Cliché Cimpenon-Bernard

jusqu'à une première fenêtre où un module à masque limite le débit envoyé dans le souterroin. Le reste est rejeté dans la Tafna.

La longueur totale du souterrain est de $11.380 \mathrm{~m}$ avec une pente moyenne de $0,7 \mathrm{~mm}$ par mètre. Deux sections types ont été adoptées

- Dans les calcaires de bonne tenue, section rectangulaire comportant un revêtement de $0,10 \mathrm{~m}$ destiné à améliorer l'écoulement hydraulique;

- Dans les zones de mauvaise tenue, section circulaire entièrement revêtue.

A son extrémité aval, le souterrain de la Tafna débouche dans un bassin de compensation : le bassin de Bou Hallou. 
Vue aérienne de la station de filtration de Bou Hallou.

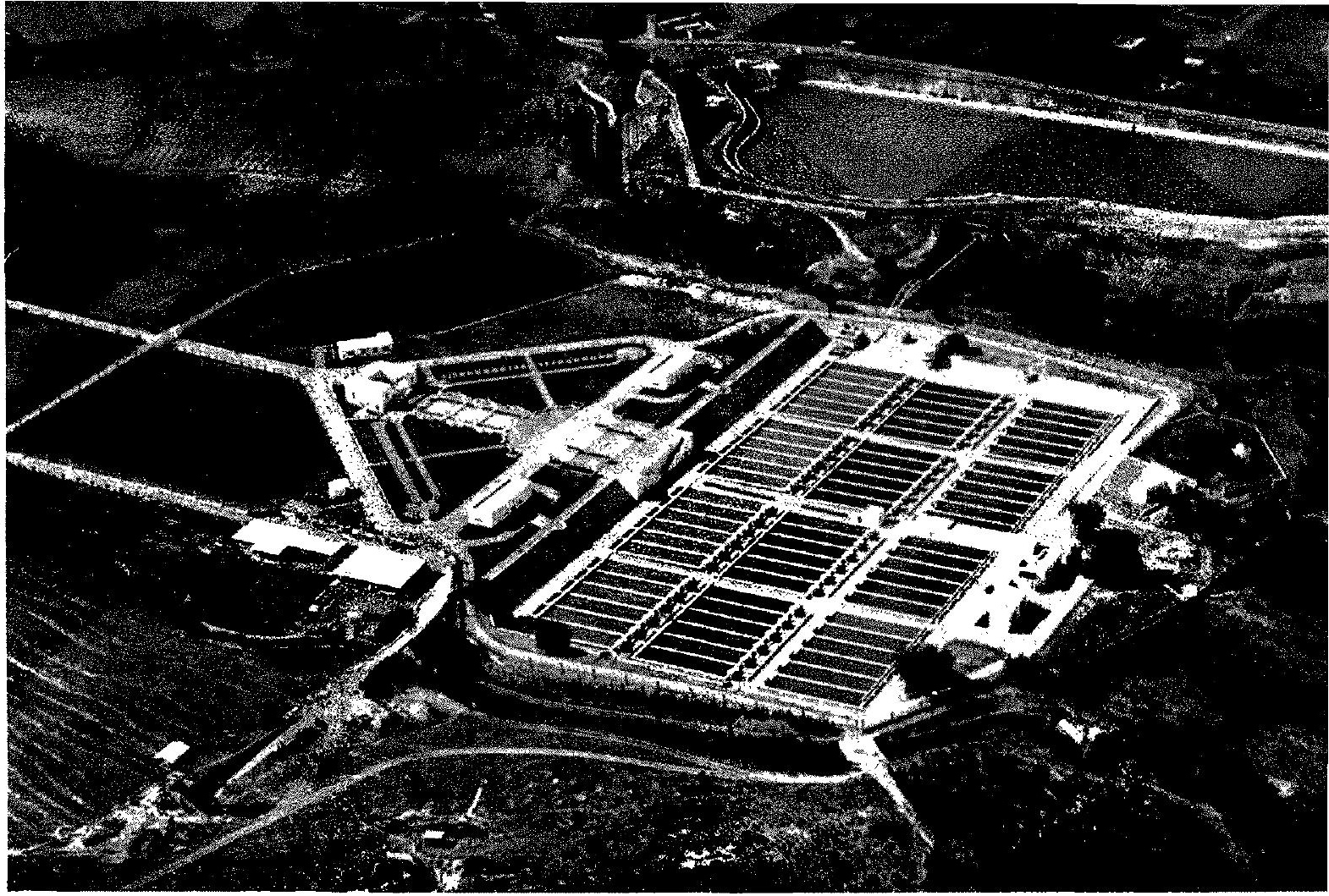

\section{BASSIN DE BOU HALLOU}

D'une capacité de $80.000 \mathrm{~m}$, Il est appelé à jouei un double rôle : d'une part, il constitue pour l'usino do pied de barrage un bassin de compensation, d'autre part, I ossure aux installations aval de la condute doran ct du réseau d'irrigations de la plaine de Marnia, une réserve d'accumulation capable d'amortir les irrógularités propres à leur exploitation.

Le fond est réglé avec une pente de l cm par mètre vers un conol collecteur central qui possède lui-mêne une pente de $4 \mathrm{~mm}$ vers l'aval. Le fond et les bajoyers sont revêtus.

Station de filtration de Bou Hallou. Détails de fonds de filtres en éléments préfabriqués.

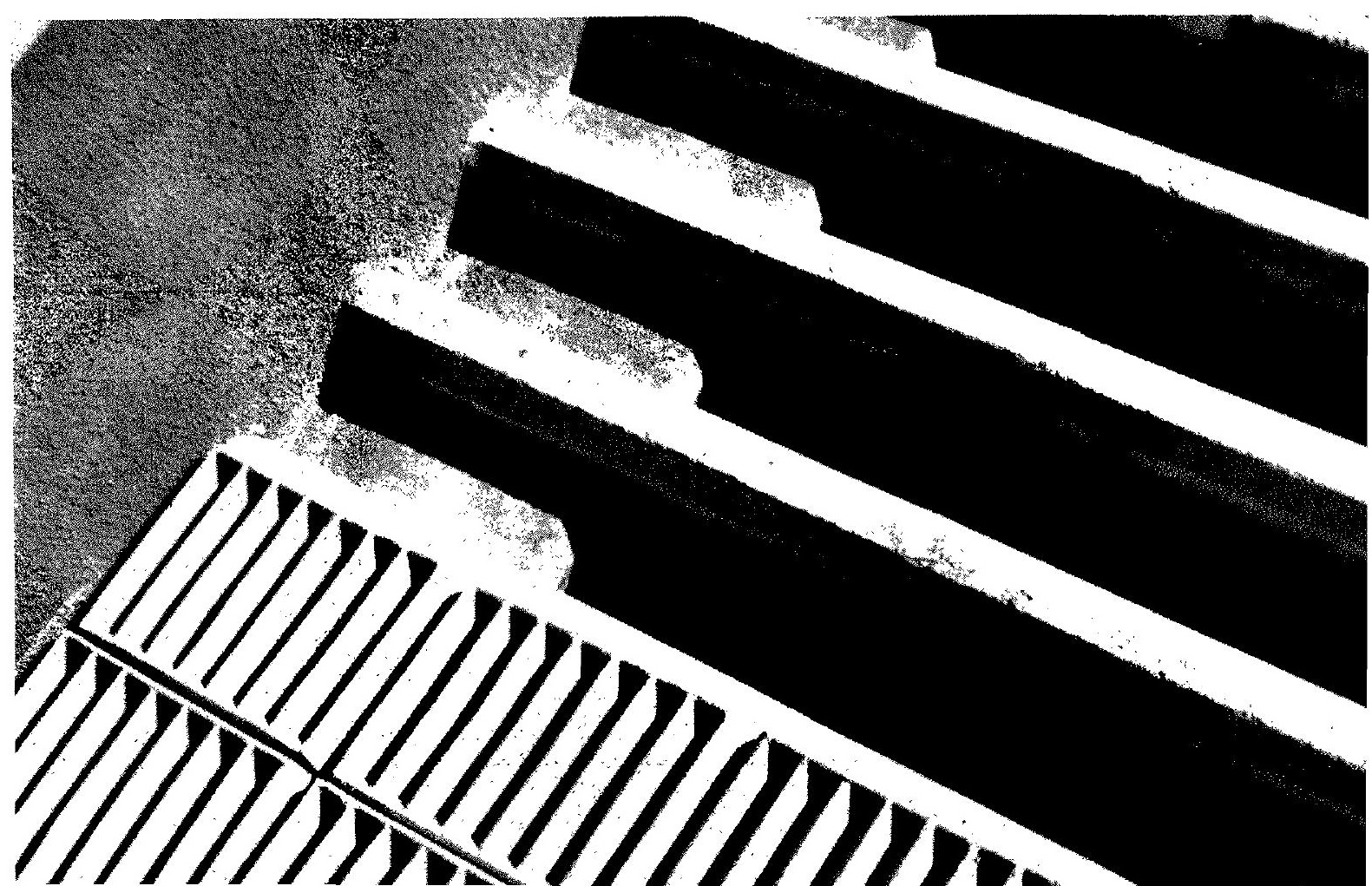



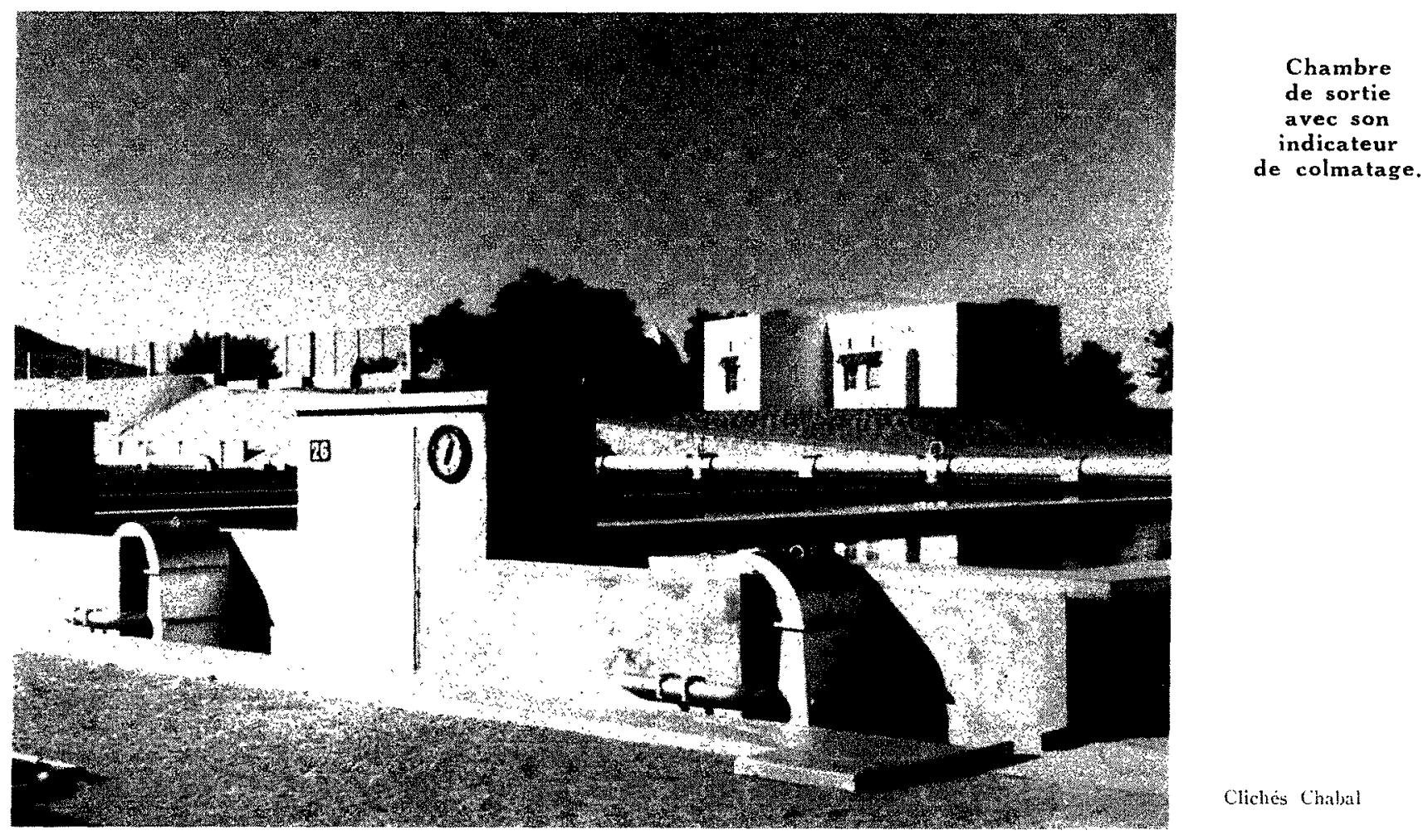

\section{STATION DE FILTRATION}

L'eau destmée à l'alimentation de la région oranaise doit évidemment subir un certain nombre de traitements destinés à lui assurer la pureté et les qualités hygiéniques requises.

Dès la sortie du bassin de compensation, en tête de la conduite, elle passe d'abord dans une station de filtration à deux étages; à l'extrémité aval, au niveau cu brise-charge $n^{\prime \prime} 8$, juste avant la distribution, l'eau est encore soumise à une stérilisation complémentaire de sécurité.

La station de filtration comprend 72 bassins standard de $153 \mathrm{~m}^{2}$ chacun, soit au total plus de $11.000 \mathrm{~m}^{2}$. On y trouve

- L'ouvrage d'alimentation à niveau variable, fonctionnant en cheminée d'équilibre et équipé des vannes régulatrices assurant la constance du plan d'eou sur les préfiltres;

- Le canal d'alimentation des préfiltres;

- Les bassins filtrants, à savoir :

- 24 préfiltres,

- 48 filtres;

Chambres de régulation hydraulique des préfiltres.

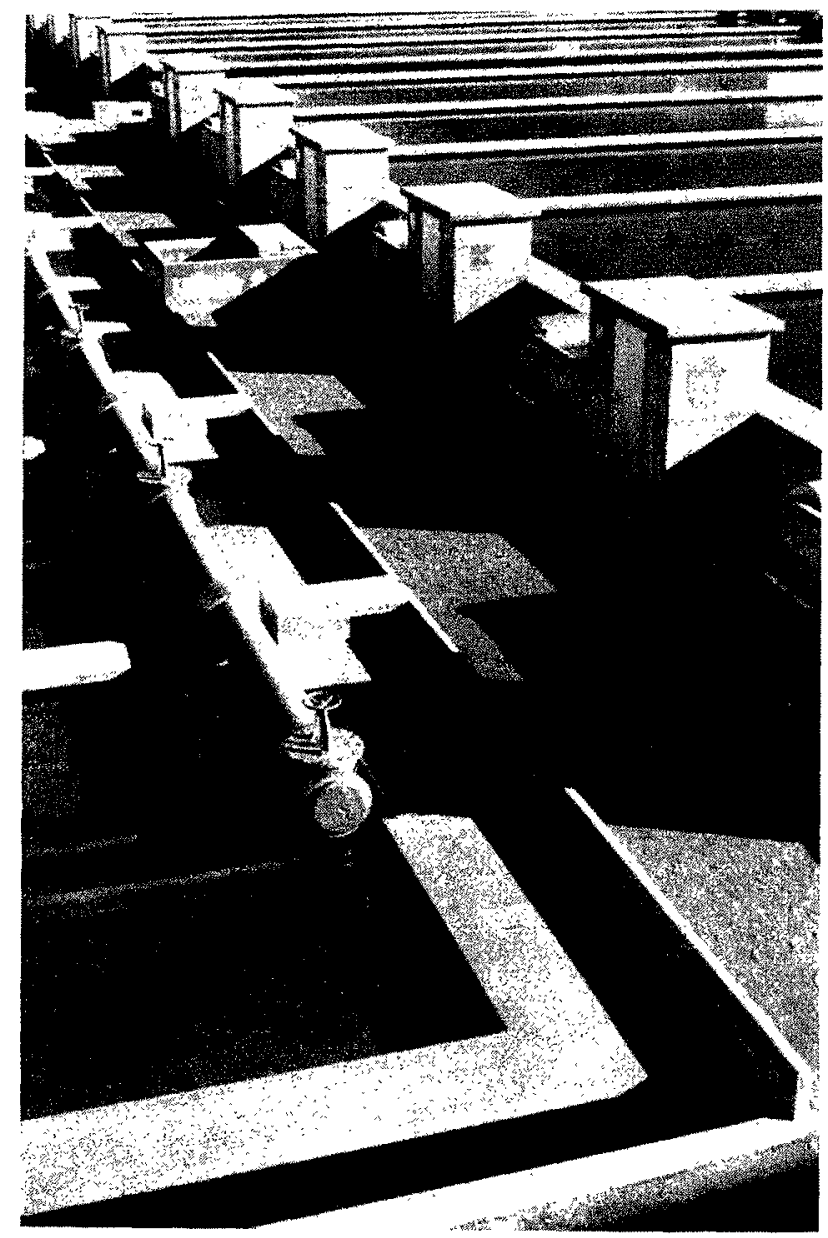



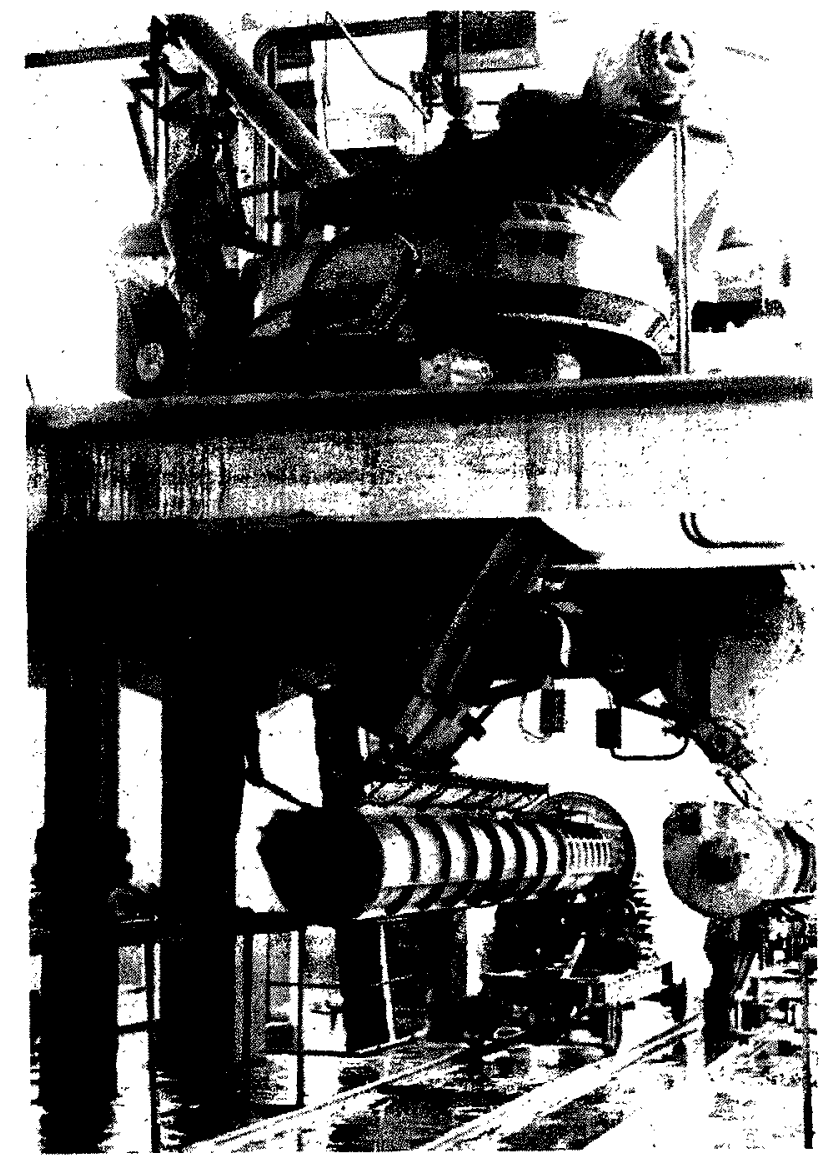

Fabrication des tuyaux :

Bétonnage primaire,

puis de haut en bas :

revêtement, frettage,

armatures mises en tension

avant centrifugation, centrifugation.

- Les blocs de régulation des bassins;

- Le réservoir d'eau filtrée $(500 \mathrm{~m}: 3)$ el lo bátiment des pompes;

- Le pavillon de présentotion des eaux trolées ef l'ouvrage de mesure de débit;

- Une station de carbonicalion;

- Les annexes.

Les innovations techniques qui caractérisent la station sont les appareils de régulation hydroulique par l'aval (en l'occurrence des vannes cylmariques à chemise autocentreuse, ou nombre de 73 : une en tête do la station, 24 à l'aval des préfiltres, 48 à l'aval des
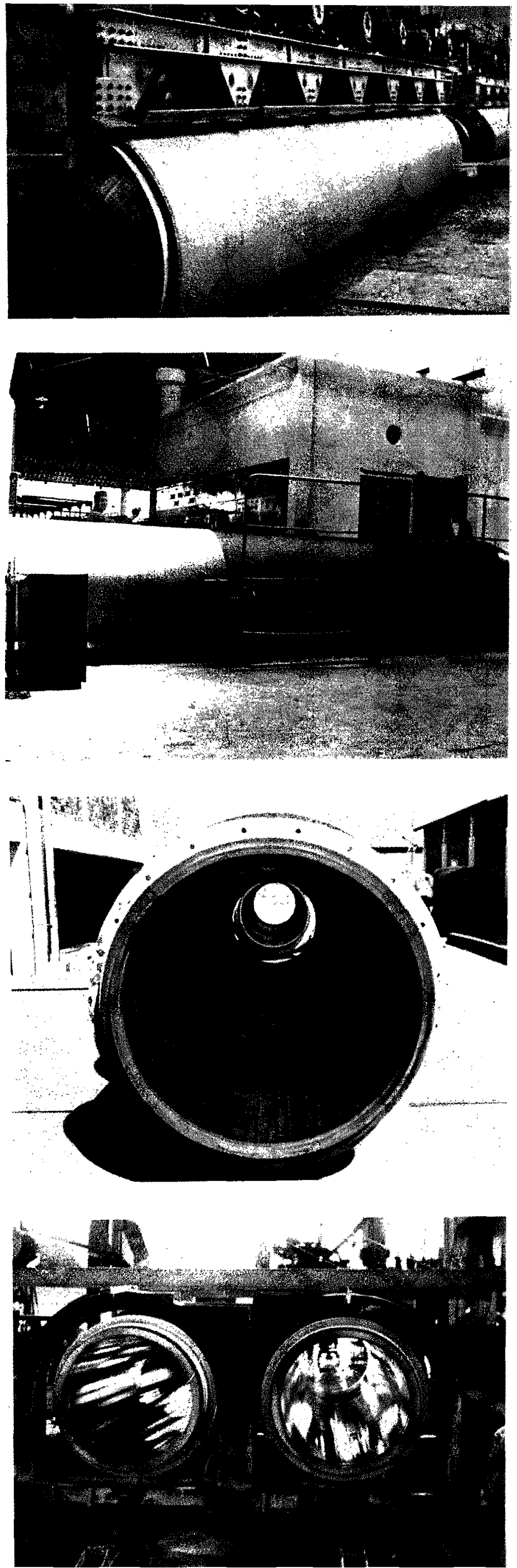


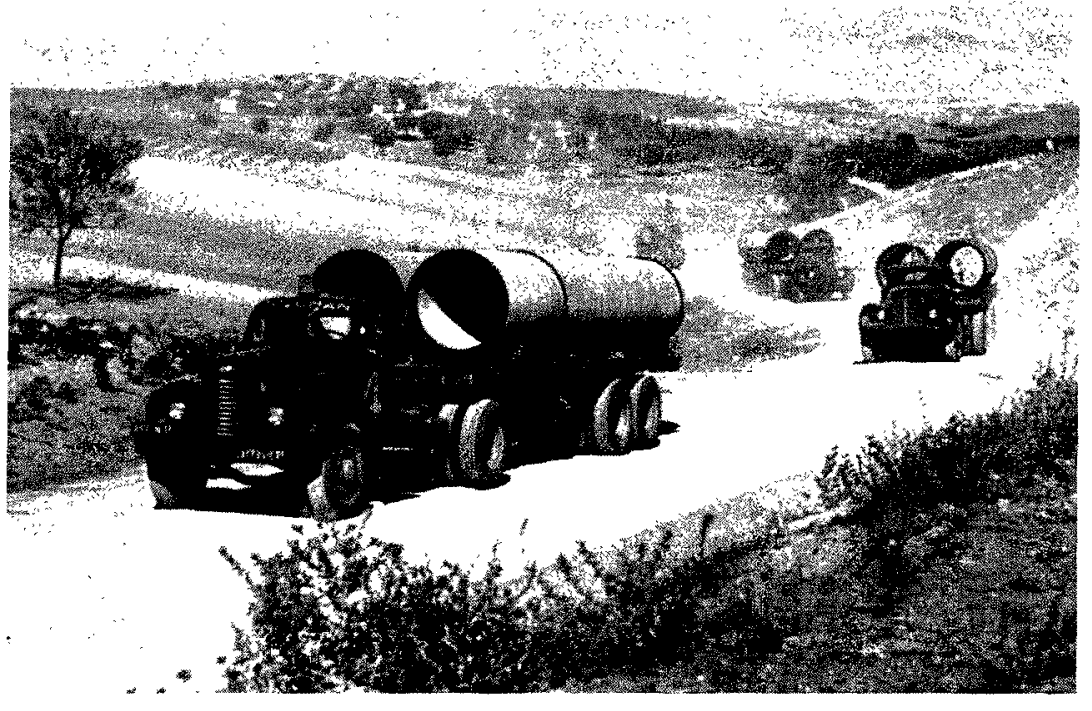

Transport de tuyaux $\varnothing 1100$.

filtres), le lavage par double siphonnage équilibré, enfin la protection de la conduite par carbonication.

\section{LA CONDUITE}

La conalisation est constituée par des éléments en béton précontraint de $7 \mathrm{~m}$ de long et $1,10 \mathrm{~m}$ de diamètre intérieur. Leur épasseur est de $8,6 \mathrm{~cm}$ et leur poids de $5.700 \mathrm{~kg}$. Dans le cas de fortes pressions, l'épaisseur atteint $12 \mathrm{~cm}$ et le poids $8.200 \mathrm{~kg}$.

La fabrication des tuyaux en béton précontraint centrifugé était réalisée à l'usine de Laferrière, située à mi-chemin entre la station de filtration de Bou Hallou et la ville d'Oran. Elle comporte 12 phases

- Montage des moules;

- Mise en place des armatures longitudinales;

- Fabrication du béton;

- Centrifugation du béton primaire;
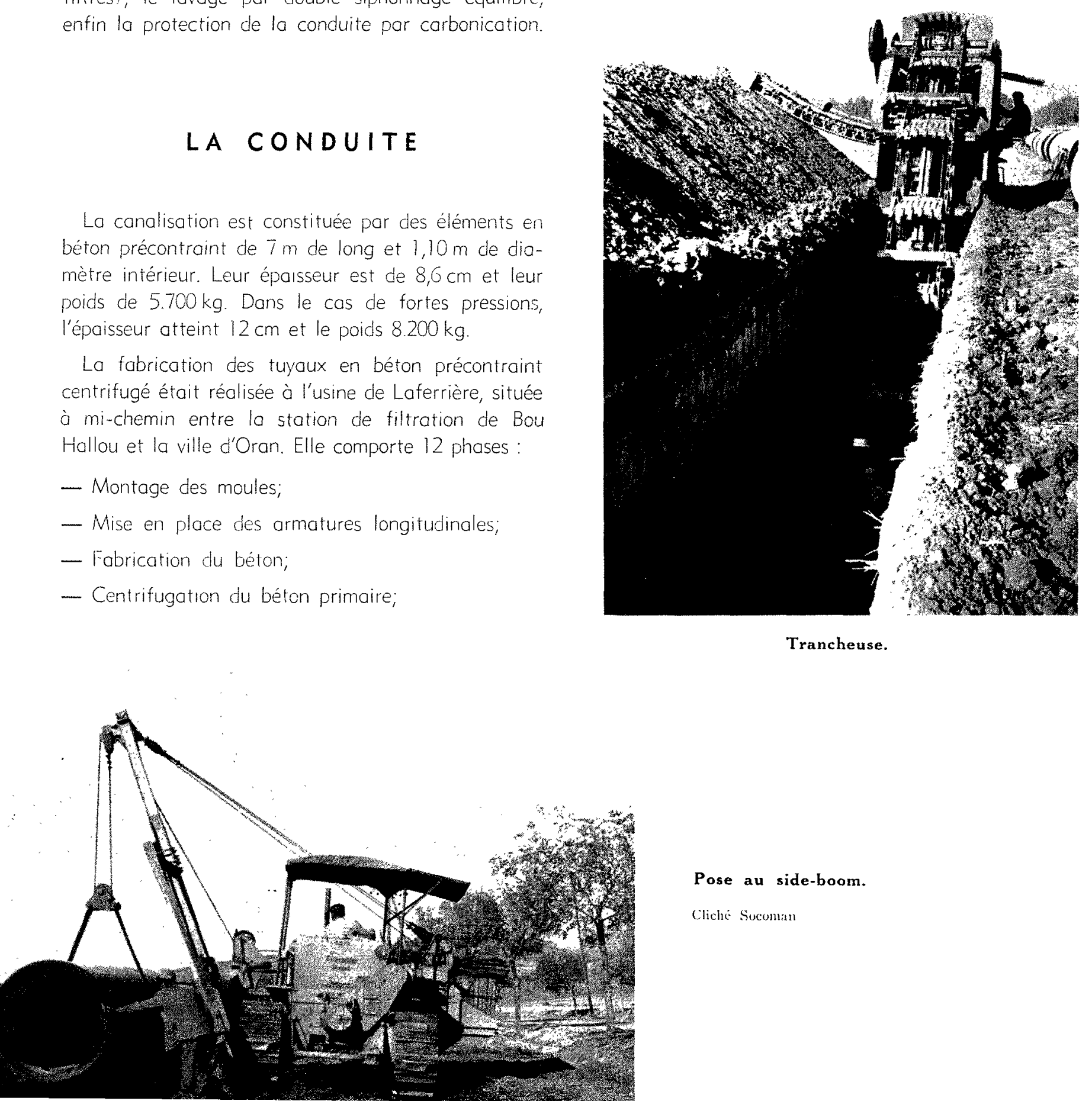

Pose au side-boom.

Clichí Sucoman 

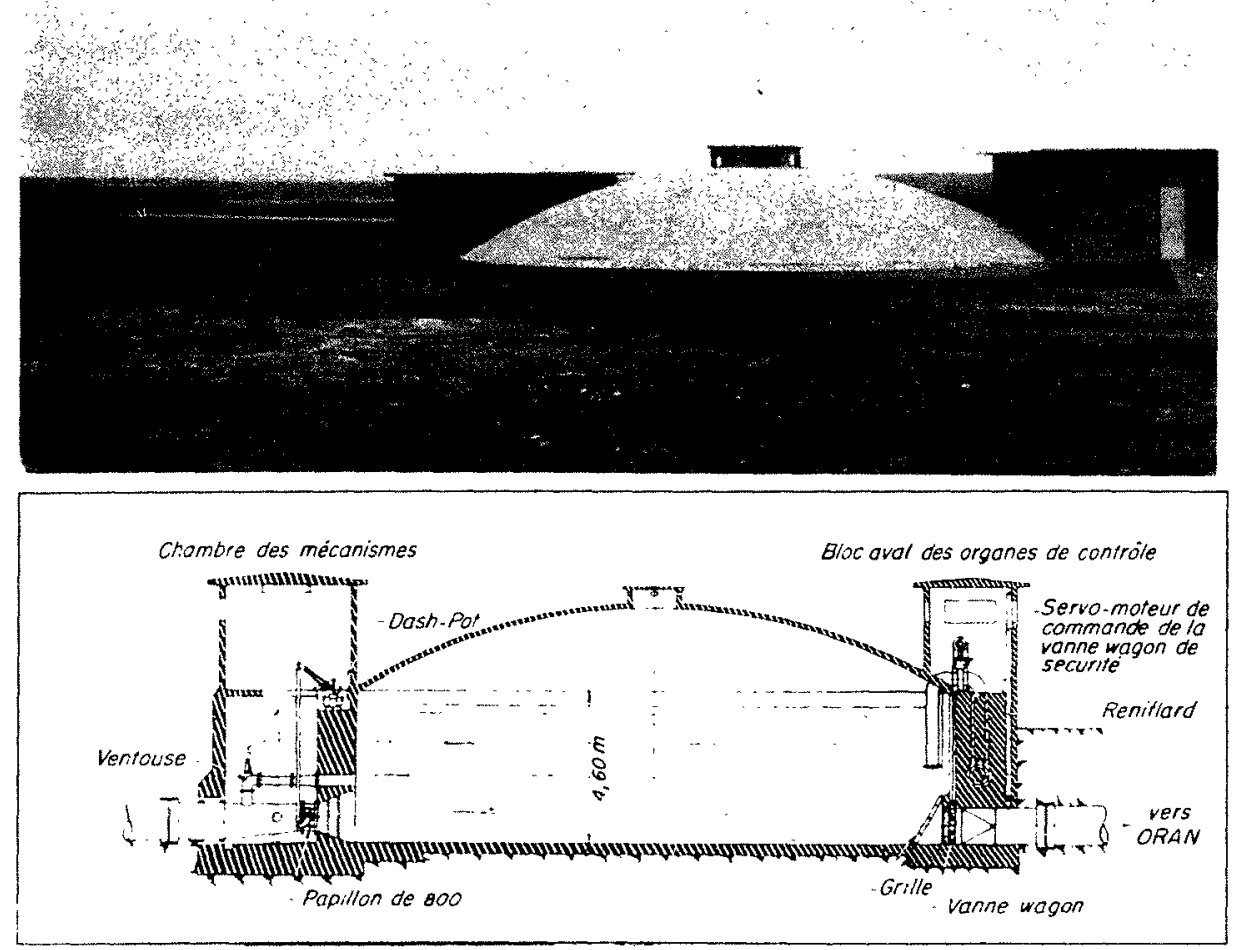

Chambre de rupture intermédiaire. Elévation

\author{
De haut en bas : \\ Vanne établissant \\ un niveau constant \\ dans le canal d'Andersson \\ faisant suite au BC VIII. \\ et
}

Timonerie et dash-pots du papillon principal $(\varnothing 800)$ d'un brise-charge.
- Etuvage du tuyau primaire;

- Démontage du tuyau primaire;

- Humidification du tuyau primaire;

- Frettoge;

- Essais;

- Mise en ploce du revêtement;

- Etuvage du revêtement;

- Humidification du revêtement.

Après avoir été stockés à la sortie de l'humidificafion secondaire, les fuyaux étaient amenés à pied d'ceuvre par comions.

Afin de protéger la conduile contre les variations de température, contre les pluies torrentielles et les glissements de terroin, elle a été enterrée sur tout son trajet, souf bien entendu au franchissement des ruisseaux et rivières. L'importance des terrassements $1800.000 \mathrm{~m}^{3}$ pour une tranchée de $1,60 \mathrm{~m}$ de large $\epsilon t$ 3 à $4 \mathrm{~m}$ de profondeur) a conduit à les mécaniser au maximum. Sur les ouvrages d'ort, lo conduite est supportée par des berceaux en béton à roison de deux par élément.

Au moment de la pose, chaque élément était soumis à une épreuve de mise en pression; ces essais furent repris ò la mise en eau des divers éléments de la conduite, cette fois par longs tronçons.
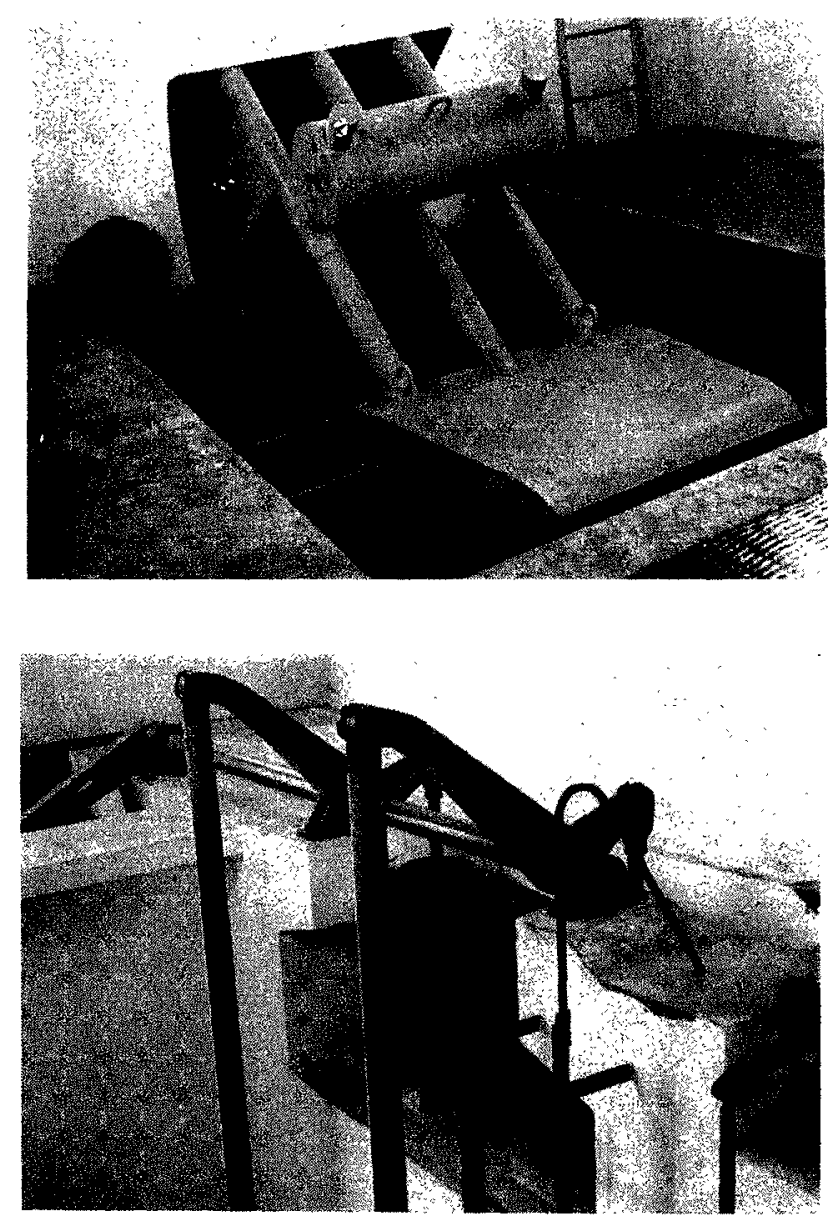
Brise-charge $n " 8$. Usine de chloramination.

Les ouvrages hydrauliques les plus mportants le long de la conduite sont les brise-charges, au nombre de 7 , situés aux points hauts. Ils comprennent trois parties distinctes:

- Lo chambre des mécanismes groupant les organes de réglage automatique de débit, convenoblement ćquipés d'appareils de sécurité, en ve d'éviter toutes manceuvres intempestives dongereuses;

- La rotonde centrale à plan d'eau réglé, où est dissipée l'énergie libérée par la rupture de charge Elle permet à la conduite de faire face a toutes variations de la demande sans oscillations ni pertes d'eau;

- Le bloc aral contenant les organes de contrôle et de sécurité protégeant le tronçon faisant suite au brise-charge
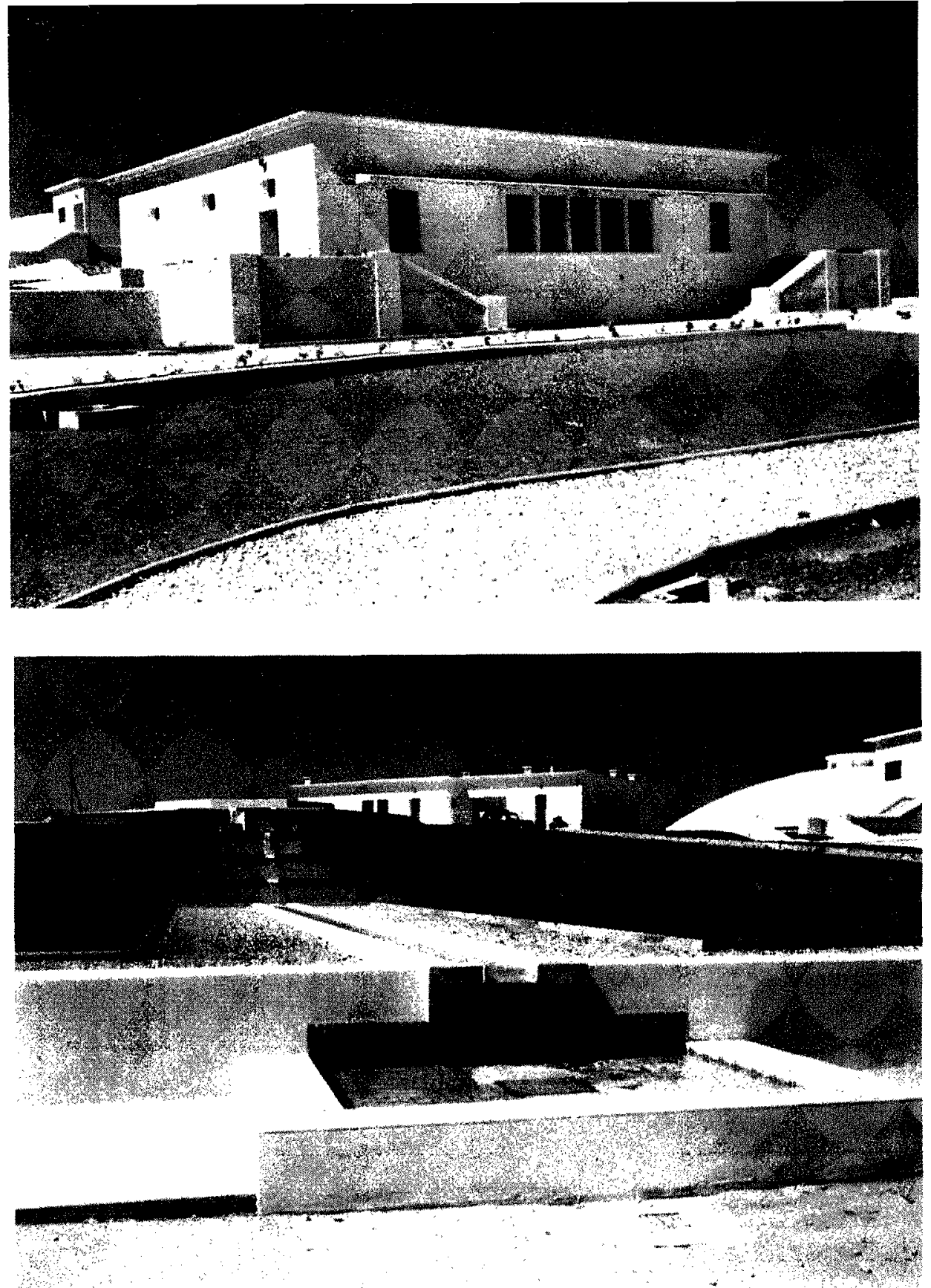

Présentation architecturale de l'escalier d'eau.

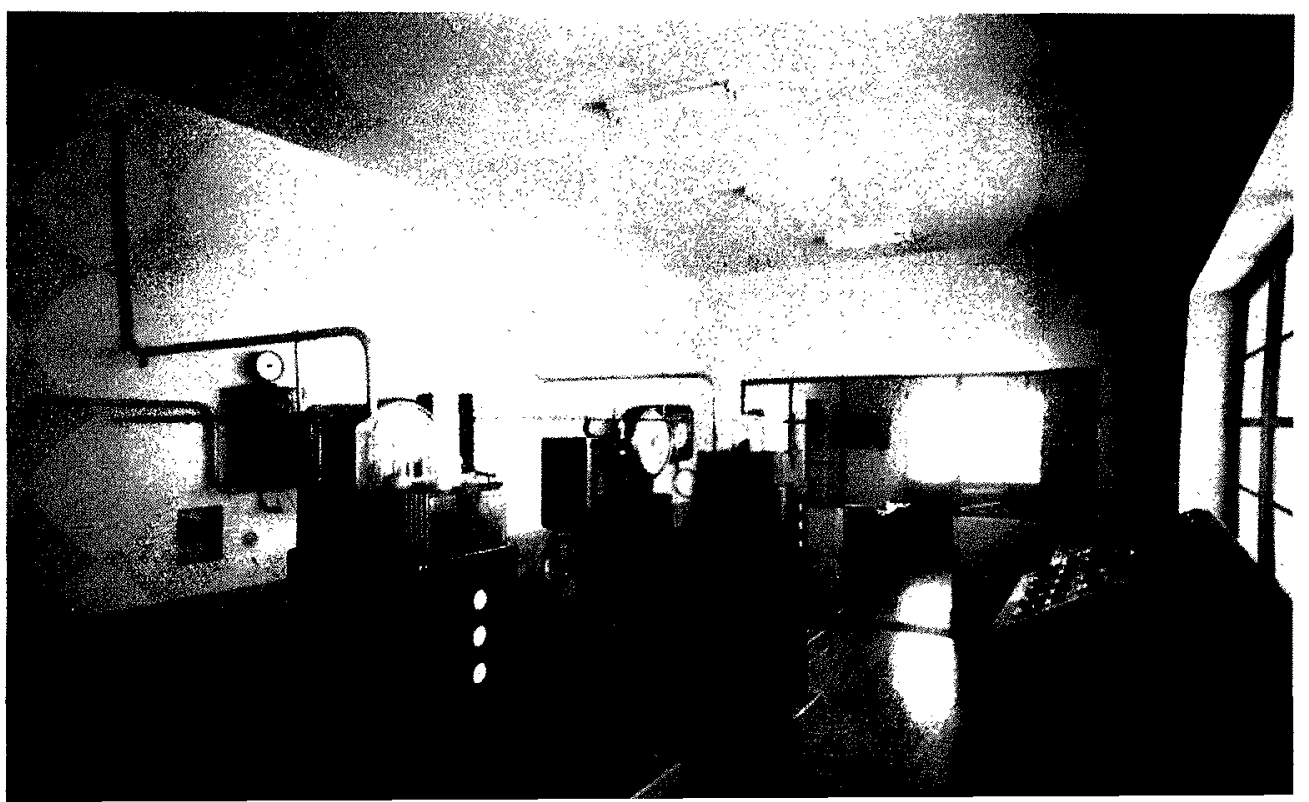

Salle de chloramination automatique. 


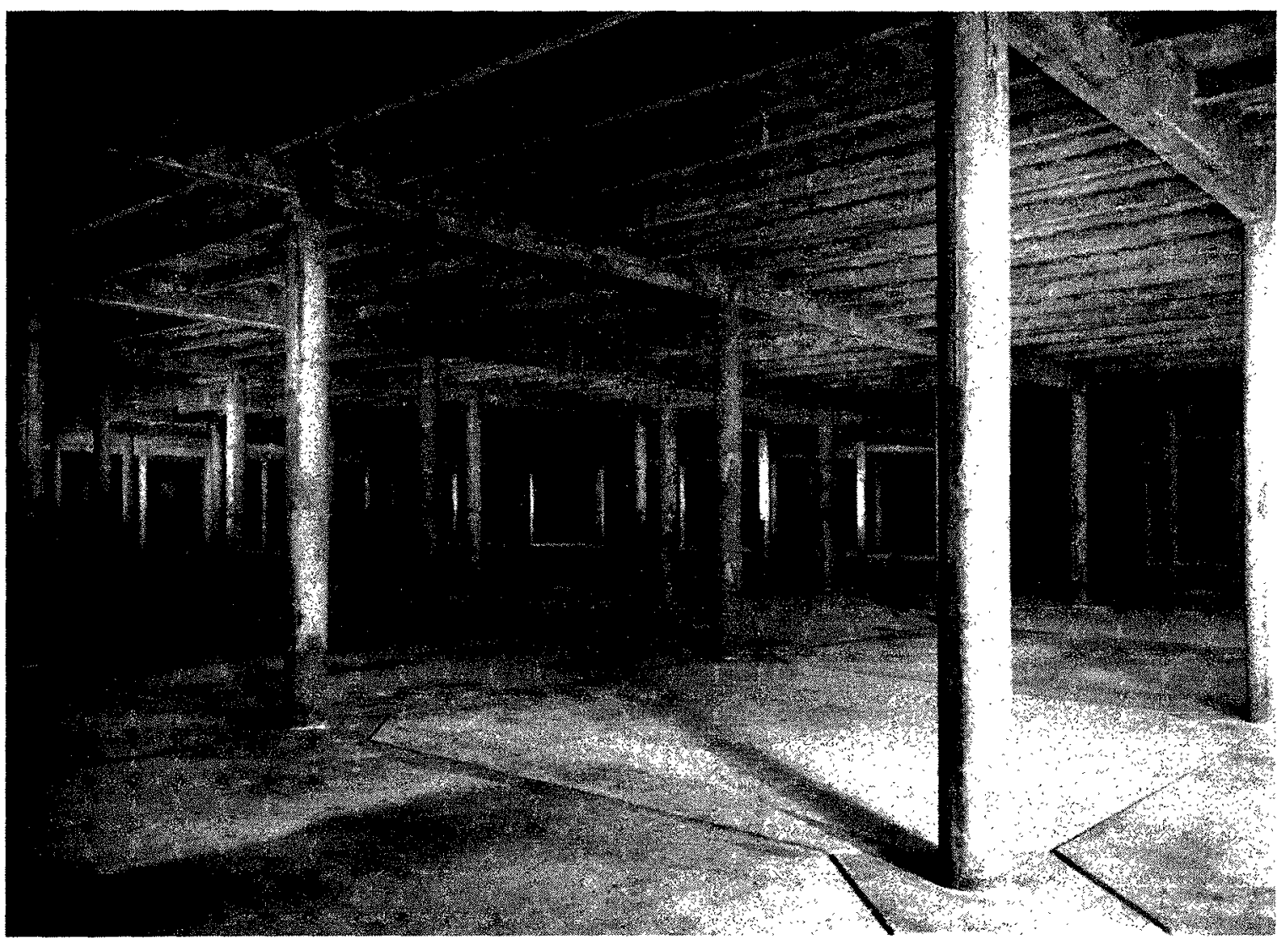

Cliché Entreprise Nord-Africaine de Constructions

Réservoir de $10000 \mathrm{~m}^{3}$ de la ville d'Oran d'Aïn Blida.

Ainsi que nous l'avons déjà signalé, le brise-charge $n^{*} 8$, en fin de canalisation, comprend également la station de stérilisation des eaux.

Trois cheminées d'équilibre composées d'un réservoir de $80 \mathrm{~m}^{3}$ assurent, concurremment avec les brisecharges, la régularité de l'écoulement dans la conduite.

En dehors de ces ouvrages, chacun des sommets du profil en long comporte une ventouse située dans une chambre en maçonnerie, tandis qu'à chaque point bas est installée une vanne de vidange. 